\title{
Valoración de la calidad de vida relacionada con la salud en la enfermedad renal crónica terminal mediante un cuestionario de resultados percibidos por los pacientes
}

\author{
Mireia Adell Lleixà̀,2, Lina Casadó Marin¹, Jeroni Andújar Solé2, Elena Solà Miravete ${ }^{1}$, Estrella Martínez \\ Segura' ${ }^{1}$,Teresa Salvadó Usach ${ }^{1}$ \\ ${ }^{1}$ Facultad de Enfermería. Universidad Rovira y Virgili. Campus Terres de I'Ebre. Tortosa. ${ }^{2}$ Unidad Nefrológica. \\ Complejo asistencial de la Santa Creu Jesús-Tortosa, Tarragona. España
}

\section{Resumen}

Objetivos: Valorar la Calidad de Vida Relacionada con la Salud de los pacientes con Enfermedad Renal Crónica Terminal e identificar las diferencias más significativas según el sexo y tratamiento recibido.

Material y métodos: Estudio observacional, analítico y transversal; realizado a pacientes en tratamiento con Hemodiálisis de Alto Flujo o Hemodiafiltración "en línea". Realizamos estadística descriptiva e inferencial y se consideraron significativos aquellos resultados en que el grado de significación resultó igual o inferior al $5 \%(p \leq 0.05)$.

Resultados: Obtuvimos diferencias estadísticamente significativas en: valores de albúmina sérica según la técnica de diálisis y dosis de tratamiento adecuado $(\mathrm{Kt} / \mathrm{V})$ según el sexo.

En las dimensiones del test de calidad de vida: síntomas/problemas, situación laboral, función sexual, función física, dolor y vitalidad (según sexo). Y según la técnica en: sueño, actitud del personal de diálisis y función física.

En el análisis multivariante, las únicas variables clínicas y sociodemográficas que resultaron ser predictores significativos en algunas de las dimensiones de las es-

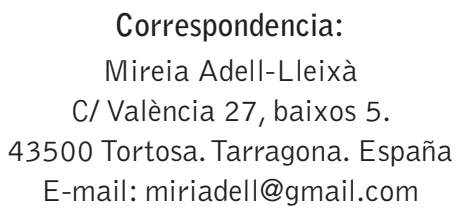

calas KDQ0L-SF fueron el sexo, el nivel de estudios y la técnica de diálisis.

Conclusiones: Las variables sociodemográficas, analíticas, el sexo y técnicas de diálisis influyen sobre la percepción de la calidad de vida de los pacientes con enfermedad renal crónica terminal.

PALABRAS CLAVE

- CALIDAD DE VIDA RELACIONADA CON LA SALUD

- HEMODIÁLISIS

- HEMODIAFILTRACIÓN ON LINE

- KDQOL-SF

Assessment of health-related quality of life in chronic end-stage renal disease using a questionnaire of patient-perceived outcomes

\section{Abstract}

Objective: Evaluate the health-related quality of life for patiens with end-stage renal disease and identify the most significant differences depending on gender and treatment received.

Methods and Materials: Observational, analytical and cross-sectional study (second semester 2014) carried out on patiens having high-flux hemodialysis or On-line hemodiafiltration treatment. We did descriptive and inferential statistics and the results with signification grades equal or less than $5 \%(p<0.05)$ were regarded as significant. 


\section{Results:}

Significant differences in:

Albumin values according to the dialysis technique and appropriate treatment doses (Kt/V) depending on gender.

Quality of life test dimensions: Symptoms/problems, employment status, sexual function, physical activity, pain and vitality (depending on gender) and according to technique with sleep, staff attitude and physical activity.

In the multi-variable analysis; gender, level of education and dialysis technique were the only clinical and sociodemographic variables which ended up being reliable predictors in some of the dimensions of the scales KDQOL-SF.

Conclusions: The socio-demographic variables, analytical, gender and dialysis techniques have influence on renal disease patient's perception.

\section{KEYWORDS}

- HEALTH-RELATED QUALITY OF LIFE

- HEMODYALISIS

- ON LINE HEMODIAFILTRATION

- KDQOL-SF

\section{Introducción}

El aumento de la esperanza de vida ha dado como resultado una elevada prevalencia de enfermedades crónicas en nuestra sociedad, propiciando así, a la evolución del concepto de Calidad de Vida Relacionada con la Salud (CVRS) y al modelo de atención de dichas enfermedades. Aunque su interés ya existía desde la época de la Antigua Grecia, no será hasta finales del siglo anterior y principios de éste cuando se produzcan los cambios más relevantes en el propio concepto de CVRS y el incremento de su estudio dentro del campo de las ciencias de la salud ${ }^{1}$.

El interés por su estudio coge impulso en el momento en que las investigaciones de las enfermedades crónicas dirigen sus avances terapéuticos, no tan solo en conseguir una mayor supervivencia sino, en mejorar la calidad de vida y promover el bienestar de dichas personas $^{2}$. La incorporación de criterios subjetivos en la evaluación de los distintos procesos de salud como la creación de instrumentos de valoración como los Cuestionarios de Resultados Percibidos por los pacientes (PRO-Patient Reported Outcomes) que tienen como eje central la percepción directa de la CVRS de la persona sin interpretación alguna de las respuestas más que la de la misma, ayudará a dar un giro al modelo biomédico clásico, acercándonos a un modelo más holístico e integral de salud³.

En 1994, la Organización Mundial de la Salud (OMS) definió el término CVRS como: "La percepción del individuo de su posición en la vida en el contexto de la cultura y sistema de valores en los que vive y en relación con sus objetivos, expectativas, estándares y preocupaciones. Además, se caracteriza por ser: Subjetiva, multidimensional, variable en el tiempo e incluir sentimientos positivos y negativos ${ }^{4}$.

Por tanto, la percepción del bienestar de cada persona depende de sus propios valores, creencias, contexto cultural e historia de vida personal. De ahí que, personas con circunstancias clínicas similares, vivan situaciones diferentes ${ }^{5}$.

Según los resultados del estudio EPIRCE (Epidemiología de la Insuficiencia Renal Crónica en España), la prevalencia de la Enfermedad Renal Crónica (ERC) en nuestro país se estima en un $9,24 \%$ en la población adulta y, de las personas que reciben Tratamiento Renal Sustitutivo (TRS), alrededor de un $46 \%$ reciben tratamiento en Hemodiálisis (HD) en alguna de sus modalidades. Teniendo en cuento los resultados anteriores, podemos decir que la ERC representa un importante problema de salud pública en nuestro país, debido a su prevalencia, elevada morbimortalidad e importante gasto económico que supone su detección, control y tratamiento ${ }^{6}$.

A pesar de los avances médicos y tecnológicos en la mejora de las técnicas de depuración extracorpórea, la ERC y su tratamiento sigue ocasionando cambios físicos, psicológicos y sociales a las personas que la padecen ${ }^{7}$. La adaptación favorable o no a estos cambios dependerá en gran parte de las estrategias que la propia persona sea capaz de desarrollar junto a su familia y al equipo multidisciplinar que le atiende ${ }^{8}$.

La población con ERC presenta una calidad de vida inferior a la del resto de la población en general'. En la bibliografía ${ }^{10}$, observamos que las personas con ERC presentan un deterioro de la CVRS des del momento del diagnóstico de la enfermedad y que se 
acentúa al empezar en programa de diálisis. Distintos estudios $^{11-13}$ demuestran que la baja CVRS favorece al aumento de la mortalidad de las personas en diálisis. De ahí, la necesidad de incorporar indicadores de calidad en el seguimiento clínico de dicha población, ya que la relación que se establece entre CVRS y morbimortalidad es muy estrecha ${ }^{14}$.

Se asocian distintas variables que influyen en la CVRS de las personas con ERC, podemos destacar: Variables clínicas, sociodemográficas, técnica de diálisis utilizada, estado cognitivo-emocional, al igual que el entorno, la familia, las creencias, la cultura a la cual pertenece la persona y las experiencias vividas $^{15-20}$.

Referente a las distintas técnicas utilizadas en el tratamiento de la Enfermedad Renal Crónica Terminal (ERCT) o estadio $V$ de la ERC, encontramos cierta discrepancia en la literatura. Por un lado, encontramos estudios recientes que certifican las ventajas de la Hemodiafiltración "On Line" (HDF-OL) respecto a la Hemodiálisis de Alto Flujo (HD-AF) ${ }^{21}$ y estudios que no encuentran diferencias significativas entre la HDF-OL y la HD-AF ${ }^{22-23}$, a no ser que estén asociadas a elevados volúmenes de convección ( $>21 \mathrm{~L}$ ).

Por todo ello, y con la hipótesis de trabajo de que los pacientes en HD que reciben tratamiento con HDFOL post dilución presentan mejores resultados clínicos y perciben una CVRS superior a las personas que reciben tratamiento con HD-AF, nos planteamos el siguiente objetivo principal:

Valorar la CVRS de los pacientes en HD e identificar las diferencias más significativas en la forma de percibir dicha CVRS según el sexo y el tratamiento recibido (HD-AF o HDF-OL), y como objetivo secundario evaluar la influencia de los parámetros analíticos y variables sociodemográficas sobre la CVRS de la población de estudio.

\section{Material y métodos:}

Se ha llevado a cabo un estudio observacional, analítico y de corte transversal, durante el segundo semestre de 2014. El ámbito de estudio fue la Unidad de Asistencia Nefrológica del Hospital de la Santa Creu de Jesús-Tortosa, la cual da cobertura sanitaria nefrológica a todas las personas con ERC de las 4 comarcas de las "Terres de I'Ebre": Baix Ebre, Montsià, Terra Alta y Ribera d'Ebre.

\section{Población y muestra:}

La población objetivo de este estudio fueron los pacientes con ERCT de nuestra unidad asistencial que estuvieran recibiendo tratamiento con HD-AF o HDF-OL durante el período de estudio y la población accesible fueron todas aquellas personas que cumplieron los criterios de inclusión determinados para la investigación. Se realizó un muestreo de tipo no probabilístico estratégico, utilizando unos criterios subjetivos para la selección de las personas que formaron parte de las muestras a estudio.

De las 104 pacientes que recibían tratamiento en nuestro centro, en el momento en que realizamos el estudio, excluimos 60 por no cumplir los criterios de inclusión en el momento de la selección de las muestras. Se seleccionaron dos muestras: una muestra de 30 pacientes que recibían tratamiento con HD-AF y otra muestra de 14 pacientes que recibían tratamiento con HDF-OL (en este caso era la totalidad de los pacientes que recibían tratamiento con HDF-OL en el momento de la recogida de datos).

\section{Criterios de inclusión:}

Aceptar participar en el estudio, edad $>18$ años, pertenecer a un programa de crónicos desde al menos 3 meses, dializarse con la misma técnica durante los 3 últimos meses (HDF-OL o HD-AF), disponer de un buen acceso vascular (AV)/ flujo de sangre (Qb): $>350 \mathrm{mlx}^{\prime}$, dializarse con un filtro de alta permeabilidad ( Kuf $>40 \mathrm{~mL} / \mathrm{h} /$ $\mathrm{mmHg}$ ), no haber estado ingresado por algún proceso agudo durante los 3 últimos meses, no presentar patología psiquiátrica y tener un buen estado cognitivo para poder responder el cuestionario.

\section{Criterios de exclusión:}

La no voluntariedad de participar en el estudio, las personas con patología asociada avanzada o terminal y personas con deterioro cognitivo, según los criterios del Test de Lobo (puntuación $<23$ puntos) ${ }^{24}$.

\section{Variables a estudio:}

La CVRS como variable dependiente y como variables independientes:

Variables sociodemográficas: Edad, sexo, estado civil, nivel de estudios, situación laboral, situación económica, convivencia, comarca y lugar de residencia (núcleo urbano/ núcleo rural). 


\section{Variables clínicas:}

Técnica de diálisis (HD-AF o HDF-OL), tiempo en tratamiento (meses), tipo de acceso vascular (AV), Hemoglobina $(\mathrm{Hb})$, urea, albúmina, creatinina, sodio, potasio, calcio, fósforo, dosis eritropoyetina (EPO) semanal, $\mathrm{Pa}-$ rathormona $(\mathrm{PTHi}), \mathrm{Kt}, \mathrm{Kt} / \mathrm{v}$ y patologías asociadas: Dislipemia, Obesidad, Hipertensión Arterial (HTA), Diabetes Mellitus (DM), Insuficiencia Cardíaca (IC), Enfermedad Pulmonar Obstructiva Crónica (EPOC) y otras.

Para la evaluación de la CVRS, utilizamos la versión española adaptada por J. Alonso y grupo CALDIVA del cuestionario de Calidad de Vida en las Enfermedades Renales (Kidney Disease Quality of Life Short Form, KDQOL-SF) ${ }^{25-26}$. Se trata de un cuestionario PRO, específico para personas con ERC en programa de diálisis. Se facilitó el cuestionario para ser auto administrado en unos 15-20 minutos. Los pacientes que presentaban déficits visuales, recibieron ayuda por parte de un/a enfermero/a de la unidad para poder contestar las preguntas (el entrevistador no formaba parte del equipo de investigación). EI KDQOL-SF incluye una parte genérica de 36 ítems del cuestionario SF-36 distribuidos en 8 dimensiones de salud física y mental (función física, rol físico, dolor, salud general, bienestar emocional, rol emocional, función social y vitalidad), una parte específica para pacientes con Enfermedad Renal (ER) de 43 ítems distribuidos en 11 dimensiones (síntomas/problemas, efectos de la enfermedad renal en la vida diaria, carga de la enfermedad, situación laboral, función cognitiva, calidad de las relaciones sociales, función sexual, sueño, apoyo social, actitud del personal de diálisis, satisfacción del paciente). En cada ítem, la puntuación oscila entre 0-100. En todos los casos, a mayor puntuación mejor calidad de vida.

Los datos clínicos se obtuvieron de las historias clínicas y los datos sociodemográficos fueron recogidos por parte del equipo investigador a través de un cuestionario creado por este mismo.

\section{Análisis de los datos:}

Las variables cualitativas se describieron mediante el número y el porcentaje. Las variables cuantitativas a través de la media y de la desviación estándar.

Las relaciones entre las diferentes variables se realizaron mediante la prueba T-Student, $U$ de Mann-Whitney y de Spearman.

Para conocer las variables asociadas a la CVRS, se realizó un análisis de regresión logística para cada dimensión del KDQOL-SF, mediante el método hacia adelante, en el que se incluyeron las variables sociodemográficas y clínicas que mostraron un grado de significación $p<0,10$.

Los datos se recogieron en una hoja de cálculo Excel y fueron analizadas con el paquete estadístico informático SPSS v20.0 para Windows.

Se consideraron significativos aquellos resultados en que el grado de significación resultó igual o inferior al $5 \%(p \leq 0.05)$.

\section{Consideraciones éticas:}

Para poder realizar el estudio, previamente se solicitó autorización a la dirección del centro y se solicitó un informe de aprobación al comité ético de investigación clínica del hospital (informe favorable).

El equipo investigador manifestó por escrito que el estudio respetaría los principios básicos de la declaración de Helsinki ${ }^{27}$.

A todos los pacientes incluidos en el estudio se les entregó el documento de consentimiento informado, donde se les informaba de: La finalidad del estudio, la voluntariedad de estar en él, la preservación y confidencialidad de los datos (utilización de códigos personales, que sólo eran conocidos por el equipo investigador), al igual que de los beneficios y de los posibles riesgos potenciales que se pudieran derivar (se entregó una copia firmada del documento a las personas partícipes del estudio y otra copia para el equipo investigador).

\section{Resultados}

Formaron parte del estudio 44 pacientes ( 30 personas recibían tratamiento con HDF-AF y 14 con HDF-OL), con una edad media de $69,86 \pm 13,5$ años, en un rango entre $31-88$ años. El 31,8\% eran mujeres.

En el resultado de las variables sociodemográficas, podríamos destacar que el $90,9 \%$ del total de la muestra vivía acompañado, que el $93,2 \%$ se encontraban en situación de inactividad laboral, el 79,5\% tenían estudios y $11,4 \%$ no recibían ningún tipo de prestación económica. El 34,1\% del total de la muestra no ayudaba en las tareas domésticas del hogar, siendo la gran mayoría de este porcentaje hombres $(86,7 \%)$. (Tabla 1). 
Tabla 1. Variables sociodemográficas de hombres y mujeres en tratamiento con HD-AF y HDF-OL.

\begin{tabular}{|c|c|c|c|c|c|}
\hline Variable: & HD-AF & HDF-OL & HOMBRES & MUJERES & TOTAL \\
\hline \multicolumn{6}{|l|}{ Estado Civil } \\
\hline Con pareja N (\%) & $23(76,7)$ & $9(64,3)$ & $23(76,7)$ & $9(64,3)$ & $32(72,7)$ \\
\hline Sin pareja N (\%) & $7(23,3)$ & $5(35,7)$ & $7(23,3)$ & $5(35,7)$ & $12(32,3)$ \\
\hline \multicolumn{6}{|l|}{ Situación laboral } \\
\hline Activo N (\%) & $1(3,4)$ & $2(14,3)$ & $2(6,7)$ & $1(7,2)$ & $3(6,8)$ \\
\hline Inactivo $\mathrm{N}(\%)$ & $29(96,6)$ & $12(85,7)$ & $28(93,3)$ & $13(92,8)$ & $41(93,2)$ \\
\hline \multicolumn{6}{|l|}{ Nivel de estudios } \\
\hline Sin estudios N (\%) & $9(70,0)$ & $0(0,0)$ & $5(16,7)$ & $4(28,6)$ & $9(20,5)$ \\
\hline Con estudios N (\%) & $21(30,0)$ & $14(100,0)$ & $25(83,3)$ & $10(71,4)$ & $35(79,5)$ \\
\hline \multicolumn{6}{|l|}{ Convivencia } \\
\hline Solo/a N (\%) & $2(6,7)$ & $2(14,3)$ & $2(6,7)$ & $2(14,3)$ & $4(9,1)$ \\
\hline Acompañado/a N (\%) & $28(93,3)$ & $12(85,7)$ & $28(93,3)$ & $12(85,7)$ & $40(90,9)$ \\
\hline \multicolumn{6}{|l|}{ Lugar de residencia } \\
\hline Núcleo urbano (>2500hab.) N (\%) & $18(60,0)$ & $12(14,3)$ & $18(60,0)$ & $12(85,7)$ & $14(31,8)$ \\
\hline Núcleo rural (<2500hab.) N (\%) & $12(40,0)$ & $2(85,7)$ & $12(40,0)$ & $2(14,3)$ & $30(68,2)$ \\
\hline \multicolumn{6}{|l|}{ Prestaciones } \\
\hline Sin prestaciones N (\%) & $5(16,7)$ & $0(0,0)$ & $2(6,7)$ & $3(21,4)$ & $5(11,4)$ \\
\hline Con prestaciones N (\%) & $25(83,3)$ & $14(100,0)$ & $28(93,3)$ & $11(78,6)$ & $39(88,6)$ \\
\hline \multicolumn{6}{|l|}{ Tareas domésticas } \\
\hline Ayuda N (\%) & $18(60,0)$ & $11(78,6)$ & $17(56,7)$ & $12(85,7)$ & $29(65,9)$ \\
\hline No Ayuda N (\%) & $12(40,0)$ & $3(21,4)$ & $13(43,3)$ & $2(14,3)$ & $15(34,1)$ \\
\hline
\end{tabular}

El $88,6 \%$ de los pacientes estudiados tenían HTA, un $25 \%$ eran diabéticas y el $97,3 \%$ eran portadores de una FAVI (Fístula Arteriovenosa).

Podemos destacar que sólo el 18,2\% habían estado trasplantados alguna vez. La media del tratamiento fue de $42,6 \pm 30,6$ meses en un rango de 3,0-149,8 meses.

En el análisis de las variables clínicas, según la técnica de diálisis, encontramos resultados estadísticamente significativos en los valores de albúmina, siendo ligeramente superiores en las personas que se dializaban con HDF-OL $(4,1 \pm 0,3 \mathrm{mg} / \mathrm{dL})$ respecto a las que lo hacían con HD-AF $(3,9 \pm 0,3 \mathrm{mg} / \mathrm{dL})$, y en los valores del $\mathrm{Kt}$, $48,3 \pm 5.6$ litros en las personas que recibían tratamiento con HD-AF y 52,0 03,9 litros para los que recibían tratamiento con HDF-0L. Si tenemos en cuenta el sexo encontramos que los hombres requerían menos dosis de EPO y valores superiores de albúmina sérica, aunque no resultaron estadísticamente significativos.

Por otra parte, resultaron estadísticamente significativas las puntaciones obtenidas por las mujeres en el $\mathrm{Kt} / \mathrm{V}(1,8 \pm 0,3)$ respecto a los hombres $(1,5 \pm 0,2)$.
En el análisis de las puntuaciones del KDQOL-SF, según la técnica de diálisis, podemos destacar que, en general, obtuvieron mejores puntuaciones las personas que recibían tratamiento con $\mathrm{HDF}-\mathrm{OL}$, excepto en las escalas de: Efectos de la enfermedad renal, función sexual, actitud del personal de diálisis y satisfacción del paciente (Figura 1). Siendo estadísticamente significativos, los resultados obtenidos en las escalas del sueño (HDF-0L: $76 \pm 14,5$ vs HD-AF: $56,8 \pm 14,3$; $\mathrm{p}=0,000$ ), actitud del personal de diálisis (HDF-OL: $80,4 \pm 18,2$ vs HD-AF: $90,5 \pm 14,0 ; p=0,050$ ) y función física (HDF-OL: $75,4 \pm 22,4$ vs HD-AF: $42,1 \pm 29,4$; $p=0,010$ ).

En las puntuaciones obtenidas en la escala KDQOL-SF, según el sexo, resultaron estadísticamente significativos los siguientes resultados (Figura 2): síntomas y problemas (hombres: $82,8 \pm 9,8$ vs mujeres: $68,1 \pm 18,2 ; p=0,008$ ), situación laboral (hombres: $34,0 \pm 33,5$ vs mujeres: $53,6 \pm 23,7 ; p=0,045)$, función sexual (hombres: $54,2 \pm 34,3$ vs mujeres: $79,5 \pm 26,7$, $\mathrm{p}=0,020$ ), función física (hombres: $62,5 \pm 27,9$ vs mujeres: $31,5 \pm 28,5 ; p=0,020$ ), dolor (hombres: $74,9 \pm 26,1$ vs mujeres: $53,4 \pm 30,1 ; p=0,020$ ) y vita- 


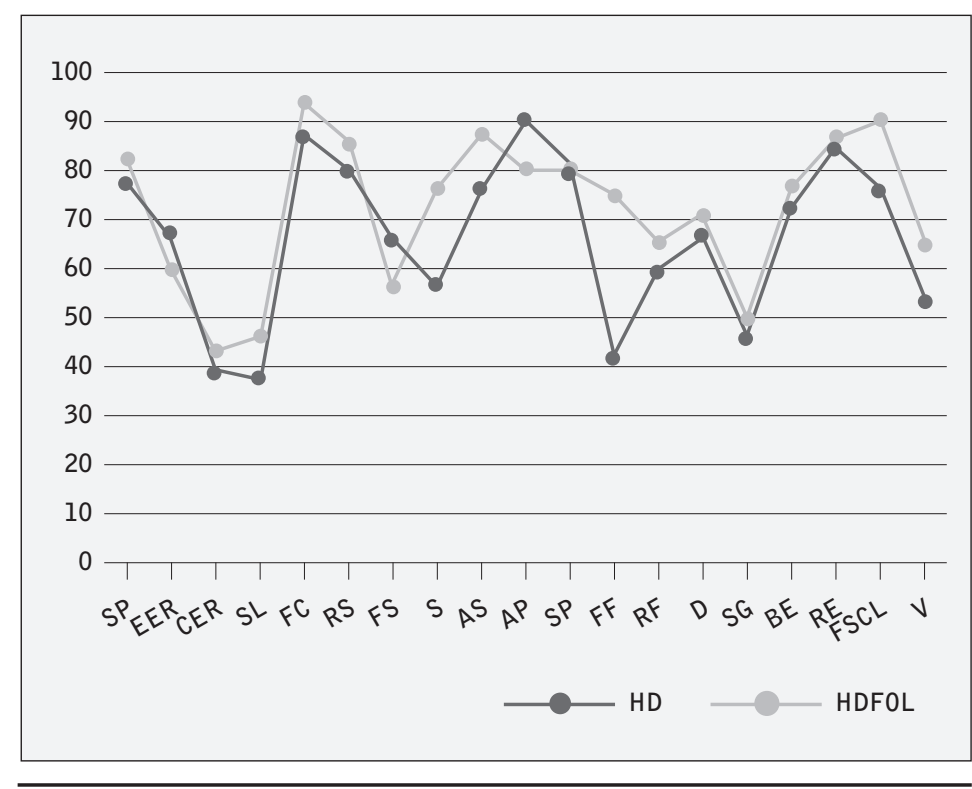

Figura 1. Puntuaciones del KDQOL-SF según la técnica de diálisis.

SP: Síntomas y Problemas; EER: Efectos de la Enfermedad Renal; CER: Carga de la Enfermedad Renal; SL: Situación Laboral; FC: Función Cognitiva; RS: Calidad de las Relaciones Sociales; FS: Función Sexual; S: Sueño; AS: Apoyo Social; AP: Actitud del Personal de Diálisis; SP: Satisfacción del Paciente; FF: Función Física; RF: Rol Físico; D: Dolor SG: Salud General; BE: Bienestar Emocional; RE: Rol Emocional; FSCL: Función Social; V: Vitalidad.

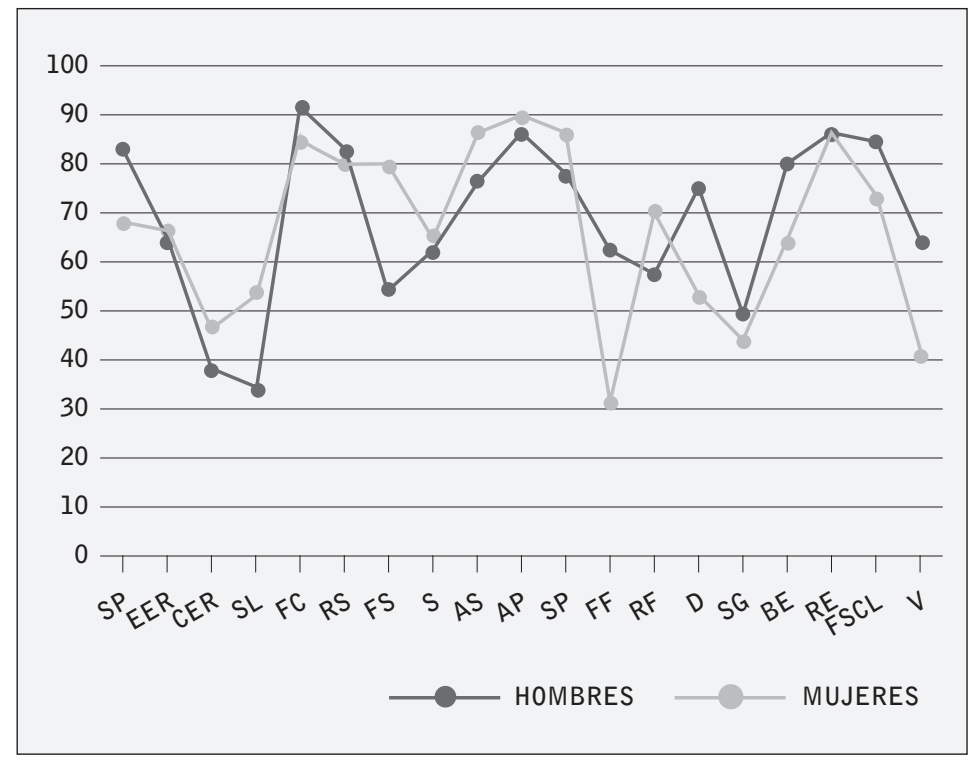

Figura 2. Puntuaciones del KDQOL-SF según el sexo.

SP: Síntomas y Problemas; EER: Efectos de la Enfermedad Renal; CER: Carga de la Enfermedad Renal; SL: Situación Laboral; FC: Función Cognitiva; RS: Calidad de las Relaciones Sociales; FS: Función Sexual; S: Sueño; AS: Apoyo Social; AP: Actitud del Personal de Diálisis; SP: Satisfacción del Paciente; FF: Función Física; RF: Rol Físico; D: Dolor; SG: Salud General; BE: Bienestar Emocional; RE: Rol Emocional; FSCL: Función Social; V: Vitalidad. lidad (hombres: $64,1 \pm 19,8$ vs mujeres: $41,5 \pm$ $27,2, p=0,013)$.

Tras analizar las diferentes variables sociodemográficas y las puntuaciones del cuestionario KDQOL-SF, los pacientes que ayudaban en las tareas domésticas, que recibían prestaciones económicas y vivían en núcleo urbano obtuvieron mejores resultados en la mayoría de ítems, aunque no resultaron estadísticamente significativos.

Sí resultaron significativos, los resultados obtenidos en las siguientes variables:

- Los pacientes que vivían solas obtenían mejores puntuaciones en las escalas de: Efectos de la enfermedad renal (solas: $86,7 \pm 12,6$ vs acompañadas: $62,6 \pm 16,3 ; p=0,007)$, sueño (solas: $80,0 \pm 11,7$ vs acompañadas: $61,1 \pm 16,4 ; p=0,031$ ) y vitalidad (solas: $85,0 \pm 7,1$ vs acompañadas $54,4 \pm 23,7$; $0,015)$.

- Los pacientes que tenían algún tipo de estudios presentaban mejores puntaciones en las escalas de: Efectos de la enfermedad renal ( $\sin$ estudios: $51,7 \pm 18,5$ vs con estudios: $68,6 \pm 15,5 ; p=0,008)$, función cognitiva (sin estudios: $80,0 \pm 18,3$ vs con estudios: $91,0 \pm 12,7 ; p=0,041$ ), sueño ( $\sin$ estudios: $51,7 \pm 11,6$ vs con estudios: $66,1 \pm 16,9 ; p=0,021)$, función física ( sin estudios: $25,6 \pm 16,2$ vs con estudios: $60,3 \pm 30,4 ; p=0,005)$, salud en general (sin estudios: $34,4 \pm 12,9$ vs con estudios: $51,2 \pm 20,5, p=0,032)$, bienestar emocional ( sin estudios: $53,8 \pm 26,8$ vs con estudios: $79,4 \pm 16,0 ; p=0,008)$, función social ( sin estudios: $56,9 \pm 34,9$ vs con estudios: $87,3 \pm 18,0 ; p=0,014$ ) y vitalidad ( $\sin$ estudios: $38,9 \pm 23,1$ vs con estudios: $62,1 \pm 22,6$; $p=0,009$ ).

- Según su situación laboral, los pacientes inactivos, presentaban mejor puntuación en la escala de rol físico (inactivas: $63,5 \pm 41,7$ vs activas: $53,3 \pm 57,7 ; p=0,041$ ).

- Las personas que no tenían pareja, obtuvieron mejores puntuaciones en las escalas de: Efectos de la enfermedad renal (sin pareja: $75,8 \pm 13,7$ vs con pareja: $60,6 \pm 17,0, p=$ 0,009 ), actitud del personal de diálisis (sin pa- 
reja: $94,8 \pm 9,9$ vs con pareja: $84,3 \pm 17,1 ; p=$ 0,052 ), rol físico (sin pareja: $83,3 \pm 30,8$ vs con pareja: $52,5 \pm 44,2 ; p=0,033$ ) y vitalidad (sin pareja: $69,3 \pm 29,1$ vs con pareja: $53,1 \pm 21,5$; $p=0,056)$.

En el análisis multivariante, las únicas variables clínicas y sociodemográficas que resultaron ser predictores significativos en algunas de las dimensiones de la escala KDQOL-SF fueron el sexo, el nivel de estudios y la técnica de diálisis (Tabla 2). El sexo masculino presenta mejores puntaciones en las escalas del dolor, bienestar emocional y vitalidad. Tener estudios favorece el obtener mejores resultados en las escalas de bienestar emocional y función social y, finalmente, recibir tratamiento con HDF-OL favorece el tener mejor CRVS en la escala de situación laboral.

\section{Discusión}

Según distintos estudios nacionales $28-29$, de los pacientes que reciben tratamiento con $\mathrm{HD}$, los hombres tienden a presentar mejores puntuaciones en las escalas del cuestionario KDQOL-SF que las mujeres, al igual que la población en general. Aunque nuestra investigación es muy parecida respecto a que las diferencias más significativas se encuentran en las puntuaciones de las escalas genéricas (función física / dolor / vitalidad) y también la obtención de mejores puntuaciones por parte de los hombres en las escalas de salud emocional, en nuestro estudio encontramos resultados que difieren en las puntaciones de las escalas específicas: Las mujeres perciben mejor su función sexual, su situación laboral y aunque presentan peor CVRS que los hombres, se encuentran más satisfechas como pacientes que ellos. Resultados que resultan similares a un estudio realizado cerca de nuestra zona ${ }^{5}$.
Al igual que en Vázquez et $\mathrm{a}^{28}$, encontramos diferencias en las variables clínicas: Los hombres presentan un mejor estado nutricional que las mujeres ${ }^{30}$ y requieren menos dosis de EPO, como los pacientes que reciben tratamiento con HDF-OL respecto a las que lo hacen con HD-AF. Resaltar que las mujeres, y de éstas las que reciben tratamiento con $\mathrm{HDF}-\mathrm{OL}$, son las que presentan mejores puntuaciones de $\mathrm{Kt} / \mathrm{V}$ y Kt.

Los pacientes que reciben tratamiento con HDFOL, en nuestro estudio, presentan una mejor CVRS respecto a los pacientes que reciben tratamiento con HD-AF, al igual que otros estudios revisados $^{31,32}$, no obstante, aceptan peor la enfermedad y están menos satisfechos con el personal de diálisis. Donde sí encontramos diferencias evidentes es a nivel clínico y de mejora de la mortalidad: Los resultados obtenidos recientemente en el estudio ESHOL ${ }^{21}$ sobre la reducción de un $30 \%$ de la mortalidad de los pacientes que reciben tratamiento con $\mathrm{HDF}-\mathrm{OL}$ versus $\mathrm{HD}$, al igual que los resultados obtenidos en los estudios CONTRAST 22 y TURKI$\mathrm{SH}^{23}$, lo ponen de manifiesto cuando el volumen de reinfusión es $>21 \mathrm{~L}$.

Los datos de nuestro estudio manifiestan que variables sociodemográficas como vivir sólo, no tener pareja y tener estudios, favorecen una mejor percepción de la CVRS.

El análisis multivariado, mostró que el hecho de ser hombre favorecía percibir menos dolor y tener más vitalidad, el tener estudios favorecía percibir una mejor salud emocional y social y recibir tratamiento con HDF-OL percibir una mejor situación laboral.

En la interpretación de los resultados de nuestro estudio, se debe tener en cuenta ciertas limitaciones

Tabla 2. Resultados del análisis de regresión logística para las dimensiones del KDQOL-SF.

\begin{tabular}{lccccc}
\hline DIMENSIÓN & V.PREDICTORA & REFERENCIA & B & IC (95\%) & p \\
\hline Dolor & Sexo & Mujer & 5,67 & $1,23-26,1$ & 0,026 \\
\hline Bienestar emocional & Estudios & Sin estudios & 19,31 & $1,55-240,48$ & 0,021 \\
& Sexo & Mujer & 23,11 & $1,48-359,81$ & 0,025 \\
\hline Función social & Estudios & Sin estudios & 9,37 & $1,75-50,22$ & 0,009 \\
\hline Vitalidad & Sexo & Mujer & 9,35 & $1,57-55,62$ & 0,014 \\
\hline Situación laboral & Técnica diálisis & Hemodiálisis & 12,44 & $1,22-126,18$ & 0,033 \\
\hline
\end{tabular}


como son, el tamaño de la muestra y el amplio rango en la edad de nuestros pacientes.

Aunque podemos decir que se cumple nuestra hipótesis de trabajo, pensamos que no podemos extrapolar toda la responsabilidad de la percepción de la CVRS a la propia enfermedad en sí y a la técnica de diálisis recibida, sino también a las propias diferencias que existen de género y de las múltiples variables psicosociales y del entorno que influyen en la percepción de la CRVS de las personas con ERCT. Para complementar estos resultados sería interesante realizar otro tipo de investigación, de tipo cualitativo, que nos puede ayudar a contestar de manera más concreta las preguntas que nos plantea estos resultados. Necesitamos obtener más información debido a la subjetividad del concepto de CVRS y a la variabilidad en la percepción de ésta, por parte de cada comunidad a estudio y así, poder ofrecer unos cuidados de enfermería más holísticos y en definitiva, de mejor calidad. La valoración de la CVRS de las personas con ERC en TRS con HD-AF - HDF-OL nos ayudará a desarrollar recursos más precisos para el abordaje de los retos que nos plantea esta enfermedad crónica compleja (como puede ser la creación de una ruta asistencial).

A la vista de nuestros resultados, podemos concluir que en los pacientes estudiados los pacientes que se dializan con HDF-OL presentan mejor CVR excepto en las escalas de efectos de la enfermedad renal, función sexual, actitud del personal de diálisis y satisfacción del paciente. Además, presentan mejor CVRS los hombres y pacientes que viven solos y los que tienen estudios.

\section{Agradecimientos}

Agradecer a todas las personas con ERCT de nuestra unidad que decidieron formar parte del estudio y a Marta Roig por ser un ejemplo de superación a seguir.

Recibido: 31 agosto 2016

Revisado: 10 septiembre 2016

Modificado: 15 octubre 2016

Aceptado: 25 octubre 2016

\section{Bibliografía}

1. Schwartzmann L. Calidad de vida relacionada con la salud: Aspectos conceptuales. Cienc. enferm. [revista en la Internet]. 2003 Dic [citado 2015 Mar 18] ; 9(2): 09-21. Disponible en: http://www.scielo. cl/scielo.php?script=sci_arttext\&pid=S071795532003000200002\&lng=es.doi:10.4067/ S071795532003000200002.

2. Casas J, Repullo JR, Pereira J. Medidas de calidad de vida relacionada con la salud. Conceptos básicos, construcción y adaptación cultural. Med Clin (Barc). 2001; 116 (20): 789-96.

3. Valderas JM, Ferrer M, Alonso J. Instrumentos de medida de la calidad de vida relacionada con la salud y de otros resultados percibidos por los pacientes. Med Clin (Barc). 2005; 125 (Supl.1): 56-60.

4. WHOQOL Group. Study protocol for the World Health Organization Project to developed a quality of life assessment instrument (WHOQOL). Qual Life Res. 1993; 2(2): 153-9.

5. Seguí Gomà $A$, Amador Peris $P$, Ramos Alcario AB. Calidad de vida en pacientes con insuficiencia renal crónica en tratamiento con diálisis. Rev Soc Esp Enferm Nefrol. 2010; 13 (3): 155-60.

6. Martínez-Castelao A, Górriz JL, Bover J, Segurade la Morena J, Cebollada J, Escalada J, et al. Documento de consenso para la detección y manejo de la enfermedad renal crónica. Nefrologia 2014; 34 (2): 243-62. doi: 10.3265/Nefrologia.pre 2014. Feb.12455

7. Rebollo P, González MP, Bobes J, Saiz P, Ortega F. Interpretación de los resultados de la calidad de vida relacionada con la salud de pacientes en terapia renal sustitutiva de la insuficiencia renal terminal. Nefrologia 2000; 20: 431-9.

8. Perales-Montilla CM, García-León A, Reyes del Paso GA. Predictores psicosociales de la calidad de vida en pacientes con insuficiencia renal crónica en tratamiento de hemodiálisis. Nefrologia 2012; 32(5): 622-30. doi: 10.3265/Nefrologia.pre2012. Jun.11447.

9. Sanz Guajardo D. El paciente actual y las opciones terapéuticas. En: Valderrábano $F$, coordinador. 
Tratado de Hemodiálisis. Barcelona: Jims/Prayma; 2004. p.31-40.

10. Pagels AA, Söderkvist BK, Medin C, Hylander $B$, Heiwe S. Health-related quality of life stages of chronic Kidney disease and at initiation of dialysis treatment. Health Qual Life Outcomes. 2012; 10:71. doi: 10.1186/1477-7525-10-71.

11. Hayashino $Y$, Fukuhara S, Akiba T, Akizawa T, Asano Y, Saito S, et al. Low health-related quality of life is associated with all-cause mortality in patients with diabetes on haemodialysis: The Japan Dialysis Outcomes and Practice Pattern Study. Diab Med. 2009, 26: 921-7. doi: 10.1111/ j.1464-5491.2009. 02800.x.

12. Gumprecht J, Zelobowska K, Gosek K, Zywiec J, Adamski M, Grzeszczak W. Quality of life among diabetic and non-diabetic patients on maintenance dialysis. Exp Clin Endocrinol Diab. 2010, 118: 205-8. doi: 10.1055/s-0029-1192023.

13. Østhus TB, Preljevic VT, Sandvik L, Leivestad T, Nordhus IH, Dammen T, et al. Heath-related quality of life in prevalent dialysis patients: Comparison between 12-items and 36-items short-form health survey. Health Qual Life Outcomes. 2012. 6;10 :46. doi: 10.1186/14777525-10-46.

14. López-Revuelta K, García-López FJ, de ÁlvaroMoreno $F$, Alonso J. Perceived mental health at the start of dialysis as a predictor of morbidity and mortality in patients with end-stage renal disease (CALVIDIA Study). Nephrol Dial Transplant. 2004; 19(9): 2347-53. PMID: 15252167 [PubMed - indexed for MEDLINE].

15. Páez $A E$, Jofré $M J$, Azpiroz $C R$, De Bortoli MA. Ansiedad y Depresión en pacientes con insuficiencia renal crónica en tratamiento de diálisis. Univ Psicol. 2009; 8 (1): 117-24.

16. Jofré R. Factores que afectan a la calidad de vida en pacientes en prediálisis, diálisis y trasplante renal. Nefrología 1999; 19 (Suppl 1): 84-90.

17. Álvarez-Ude F, Fernández-Reyes MJ, Vázquez A, Mon C, Sánchez R, Rebollo P. Sintomatología física y trastornos emocionales en pacientes en programa de hemodiálisis periódica. Nefrologia 2001; 21(2): 191-9.
18. Kimmel PL. Psychosocial factors in dialysis patients. Kidney Int. 2001; 59 (4):1599-613. PMID: 11260433 [PubMed - indexed for MEDLINE].

19. Ledón Llanes L. Enfermedades crónicas y vida cotidiana. Revista Cubana de Salud Pública 2011; 37 (4): 488-99.

20. Ottaviani AC, Nestor Souza E, De Camargo Drago N, Zazzetta de Mendiondo MS, lost Pavarini SC, de Souza Orlandi F. Esperanza y espiritualidad de pacientes renales crónicos en hemodiálisis: Un estudio de correlación. Rev. Latino-Am. Enfermagem 2014; 22(2): 248-54. doi: 10.1590/0104-1169.3323.2409.

21. Maduell F, Moreso F, Pons M, Ramos R, MoraMacià J, Carreras J, et al; ESHOL Study Group. High-efficiency postdilution online hemodiafiltration reduces all-cause mortality in hemodialysis patients. J Am Soc Nephrol 2013; 24(3): 487-97.

22. Grooteman $M P$, van den Dorpel $M A$, Bots $M L$, Penne EL, van der Weerd NC, Mazairac AH, et al; CONTRAST Investigators. Effect of online hemodiafiltration on all-cause mortality and cardiovascular outcomes. J Am Soc Nephrol 2012; 23(6): 1087-96.

23. Ercan Ok, Gulay A, Huseyin Toz, Ebru Sevinc Ok, Fatih K, Mumtaz Y, et al; Turkish Online Haemodiafiltration Study. Mortality and cardiovascular events in online haemodiafiltration (OL-HDF) compared with high flux dialysis: results fron the Turkish OL-HDF Study. Nephrol Dial Transplant 2013; 28: 109-202. doi: 10.1093/ ndt/gfs407.

24. Lobo A, Saz P, Marcos G, Día J.L, Cámara C.D.L, Ventura $T$, et al. Revalidación y normalización del Mini-Exámen Cognoscitivo (primera versión en castellano del Mini-Mental Status Examination) en la población general geriátrica. Med Clin. 1999; 112(20): 767-74.

25. García F, López K, De Álvaro F, Álvarez-U de F, Alonso J, en representación del grupo CALDIVIA. Salud percibida en pacientes que comienzan tratamiento renal sustitutivo: validación preliminar de la versión española del KDQ0L-SF. Nefrología 1998; 18 (Suppl 3): S66. 
26. Hays RD, Kallich JD, Mapes DL, Coons SJ, Carter WB. Development of the Kidney disease quality of life (KDQOL) instrument. Qual Life Res. 1994; 3: 329-38.

27. Mazzanti Di Ruggiero MA. Declaración de Helsinki, principios y valores bioéticos en juego en la investigación médica con seres humanos. Revista Colombiana de Bioética 2011; 6 (1): 12544. Disponible en: http://www.redalyc.org/articulo. oa? id=189219032009

28. Vázquez I, Valderrábano F, Fort J, Jofré R, LópezGómez JM, Moreno F, et al. En nombre del grupo cooperativo español de la calidad de vida del paciente renal de la Sociedad Española de Nefrología. Diferencias en la calidad de vida con la salud entre Hombres y mujeres en tratamiento en hemodiálisis. Nefrologia 2004; 24 (2): 167-78.

29. Gil JM, García MJ, Foronda J, Borrego JF, Sánchez MC, Pérez del Barrio $P$, et al. Calidad de vida relacionada con la salud en pacientes ancianos en hemodiálisis. Nefrologia 2003;23(6): 528-37.

30. Bel R. Valoración del estado nutricional e inflamatorio en pacientes en hemodiafiltración online: Diferencias por sexo. Nure Inv. 2016; 13(80): $1-9$.
31. Karkar A, Abdelrahman M, Locatelli F. A Randomized Trial on Health-Related Patient Satisfaction Level with High-Efficiency Online Hemodiafiltration versus High-Flux Dialysis. Blood Purif. 2015;40(1):84-91. doi: 10.1159/000381255.

32. Borrelli $S$, Minutolo $R$, De Nicola $L$, De Simone W, De Simone E, Zito B, et al. Quality of life of hemodialysis patients in Central and Southern Italy: cross-sectional comparison between Hemodiafiltration with endogenous reinfusion (HFR) and Bicarbonate Hemodialysis. G Ital Nefrol. 2016 May-Jun;33(3). pii: gin/33.3.8.

33. Rebollo-Rubio A, Morales-Asensio JM, PonsRaventos ME, Mansilla-Francisco JJ. Revisión de estudios sobre calidad de vida relacionada con la salud en la enfermedad renal crónica avanzada en España. Nefrología 2015; 35(1): 92-109. doi:10.3265/Nefrologia.pre2014. Jul.12133. 